\title{
Modulares Condition Monitoring System zur Überwachung von Fluidqualität und Partikelkontamination am Beispiel von Öl
}

\author{
E. Pignanelli ${ }^{1}, T$. Bley ${ }^{1}$, A. Schütze ${ }^{2}$ \\ ${ }^{1}$ Zentrum für Mechatronik und Automatisierungstechnik g GmbH, 66121 Saarbrücken/GER, \\ Eliseo.Pignanelli@mechatronikzentrum.de \\ ${ }^{2}$ Universität des Saarlandes/Lehrstuhl für Messtechnik, 66123 Saarbrücken/GER
}

\begin{abstract}
Zusammenfassung:
Ein miniaturisiertes mehrkanaliges Zustandsüberwachungssystem für technische Fluide wurde realisiert, um mittels nicht-dispersiver Infrarot (IR)-Absorption die Ölqualität zu bestimmen. Mit diesem System ist es möglich, mit entsprechender Anpassung der IR-Filter an verschiedene Basisfluide z.B. die Oxidation oder den Additivabbau in Schmierstoffen zu überwachen sowie eine zunehmende Wasserverunreinigung, die zu einer Verschlechterung der Fluideigenschaften führt, zu detektieren. Dieses System wurde exemplarisch bei einem Feldtest erprobt und der Verschleiß des Kühlschmierstoffes einer Schraubenpresse beurteilt. Dabei wurde eine eindeutige Korrelation zwischen verarbeitetem Detektorsignal und Belastung/Verschleiß festgestellt. Dieses System wurde um einen Partikelmonitor erweitert, der auf dem Prinzip der Lichtstreuung basiert und Informationen sowohl über die Partikelkonzentration und als auch die Partikelart (Luftblasen, Metall- oder Staubpartikel) liefert. Die Kombination beider Sensorprinzipien ermöglicht die Realisierung eines Online-Zustandsüberwachungssystems zur Verbesserung der Anlagensicherheit, z.B. für Windenergieanlagen, und zur Reduzierung der Kosten für Laboranalysen und bietet zudem eine Basis für die Selbstüberwachung des Gesamtsystems.
\end{abstract}

Key words: Infrarot, Fluidqualität, Ölzustand, Partikeldetektion, Condition Monitoring System.

\section{Motivation}

Die Einsatzdauer und der Verschleiß von technischen Fluiden sind abhängig von den Betriebsbedingungen, so dass sich eine generelle Prognose der verbleibenden Nutzungsdauer extrem schwierig gestaltet. Bei Anwendungen, in denen große Schmierstoffmengen im Einsatz sind, ist eine kontinuierliche Fluidqualitätsüberwachung wichtig, um Systemschäden frühzeitig erfassen zu können. Gewöhnlich wird zum Vorbeugen von Systemschäden entweder der Fluidwechsel unabhängig von den Fluideigenschaften in festen Zeitintervallen durchgeführt oder die verbleibende Nutzungsdauer durch die Entnahme von Fluidproben bestimmt. Die entnommenen Proben werden in speziell ausgestatteten Laboren kontrolliert. Diese Methode der Fluidüberwachung mittels Laboruntersuchungen ist jedoch zum einen sehr kostenintensiv, zum anderen verbleibt eine Unsicherheit zwischen den einzelnen Probenentnahmen. Zudem ist die Entnahme repräsentativer Proben nicht unproblematisch, da Art und Ort der Probenentnahme vor allem bei der Partikelanalyse zu stark abweichenden Ergebnissen im Labor führen kann. Für schwer zugängliche Anwendungen, zum Beispiel Offshore-Windkraftanlagen, kann eine plötzliche Verschlechterung der Fluidqualität verbunden mit schlechteren Schmiereigenschaften zu einem Anlagenschaden führen, während eine zunehmende Belastung mit Metallpartikeln ein Hinweis auf beginnende Schäden in mechanischen Komponenten ist. In beiden Fällen können größere Schäden durch frühzeitige Erkennung und passende Gegenmaßnahmen vermieden werden.

\section{Sensorsystem}

Das hier vorgestellte kombinierte Zustandsüberwachungssystem besteht aus einem Ölqualitäts- und einem Partikelsensor, die im Folgenden näher beschrieben werden.

\section{Ölqualitätssensor}

Ausgehend von der spektralen Charakterisierung von Fluiden wurde ein miniaturisiertes Messsystem entwickelt, das im Kern eine 
infrarot(IR)-transparente Siliziummesszelle besitzt. Mit diesem System ist es möglich, optische Analysen an technischen Fluiden wie z.B. Kühl-, Schmier- und Hydraulikölen durchzuführen [1]. Eine Prinzipskizze des Messsystems zur Bestimmung der Ölqualität ist in Bild 1 dargestellt.

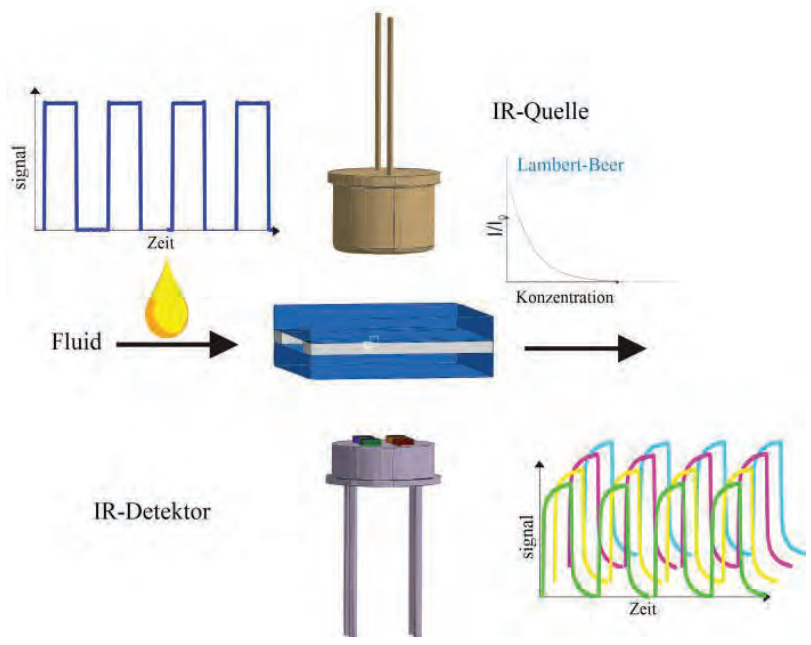

Bild 1 Funktionsprinzip des Mehrkanal-IRMesssystems zur Bestimmung der Ölqualität.

Eine kommerziell erhältliche breitbandige IRQuelle wird mit einem Rechtecksignal angesteuert. Die von der IR-Quelle emittierte thermische Schwarzkörperstrahlung strahlt durch eine IR-transparente Siliziummesszelle, die aus zwei $2 \mathrm{~mm}$ dicken Siliziumfenstern besteht. Eine Low Temperature Cofired Ceramic (LTCC)-Schicht trennt die Fenster voneinander und bildet gleichzeitig einen Fluidkanal. Mit diesem LTCC-Abstandshalter ist es möglich, einen Fluidkanal von 0,2 mm Dicke zu realisieren passend zur IR-Absorption im Medium.

Die diesem Funktionsprinzip zugrunde liegende Gesetzmäßigkeit ist das Lambert-Beersche Gesetz [2], welches den Zusammenhang beschreibt, wie stark Strahlung beim Durchgang durch ein Medium absorbiert wird. Die Absorption ist abhängig von der Schichtdicke, der Stoffkonzentration sowie vom wellenlängenabhängigen Absorptionskoeffizienten des Mediums. Die abgeschwächte Strahlung trifft anschließend auf einen vierkanaligen IRThermopile-Detektor. Jeder Kanal des Detektors besitzt einen spektral schmalbandigen Bandpassfilter; diese werden auf die charakteristischen Absorptionsbanden des zu untersuchenden Fluids adaptiert. Einer der Filter wird als Referenzkanal genutzt, um breitbandige Signaländerungen zu erfassen und zu kompensieren, die durch Änderungen in der IR-Intensität hervorgerufen werden, z.B. durch Alterung der IR-Quelle oder durch Ruß im Fluid. Die drei weiteren Wellenlängen der
Messkanäle werden auf die spektralen Bereiche abgestimmt, bei denen es in der Chemie des Fluids zu zustandsabhängigen Änderungen kommt. Anschließend werden die erfassten Signale verstärkt und mit einem FFTAlgorithmus zur Verbesserung des SignalRausch-Verhältnisses ausgewertet.

\section{Partikelsensor}

Die heutzutage standardmäßig eingesetzten Partikelsensoren, die auf dem Abschattungsprinzip beruhen, können nicht zwischen Partikeln und Luftblasen unterscheiden. Basierend auf Vorarbeiten am ZeMA [3] ermöglicht dieses Sensorsystem die Erfassung der Partikelkontamination und die Unterscheidung zwischen Luftblasen, Staub- und Metallpartikeln.

Der Partikelmonitor besteht aus einer ebenfalls gepulsten IR-Laserdiode und drei Fotodioden, die in unterschiedlichen Winkeln angeordnet sind, um Streulicht in Vorwärts- und Rückwärtsrichtung sowie das Transmissionssignal zu bestimmen (Bild 2) [4]. Je nach Verunreinigung, die den optischen Pfad der Flusszelle mit dem Fluid durchfließt, registrieren die Fotodioden unterschiedliche Streuanteile sowie eine Änderung der Transmission. Das zugrunde liegende Phänomen der Streuung von Laserlicht an Partikeln oder Luftblasen kann mit der Streutheorie beschrieben werden. Daraus resultiert eine Änderung der Strahlungsintensität in den unterschiedlichen Richtungen, die durch die in unterschiedlichen Winkeln angeordneten Fotodioden erfasst werden. In Bild 3 ist ein Schnitt durch das Partikelanalysesystem dargestellt. Für Partikel, deren Durchmesser in der gleichen Größenordnung wie die Laserwellenlänge liegt, wird die Streuung durch die MieTheorie beschrieben [5, 6]. Das von den Fotodioden empfangene Streulicht wird als Fotostrom registriert, verstärkt und mittels Transimpedanzverstärker in eine Spannung umgewandelt. Ein FFT-Algorithmus bestimmt die erste Harmonische jedes Fotodiodensignals zur Verbesserung des Signal-RauschVerhältnisses. Ausgehend vom Verhältnis zwischen Vorwärts- zu Rückwärtsstreuung sowie der Signalamplitude können Verunreinigungen wie Staub- und Metallpartikel sowie Luftblasen unterschieden werden. 


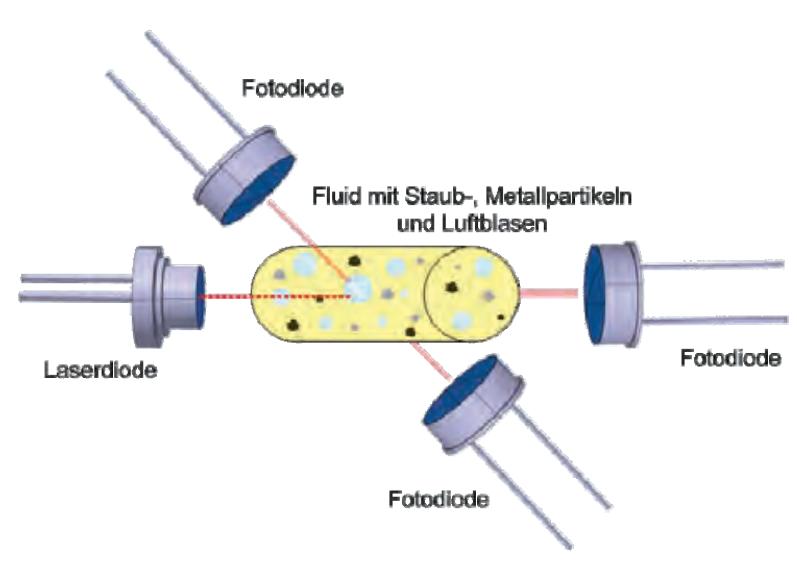

Bild 2 Funktionsprinzip des Partikelanalysesystems.

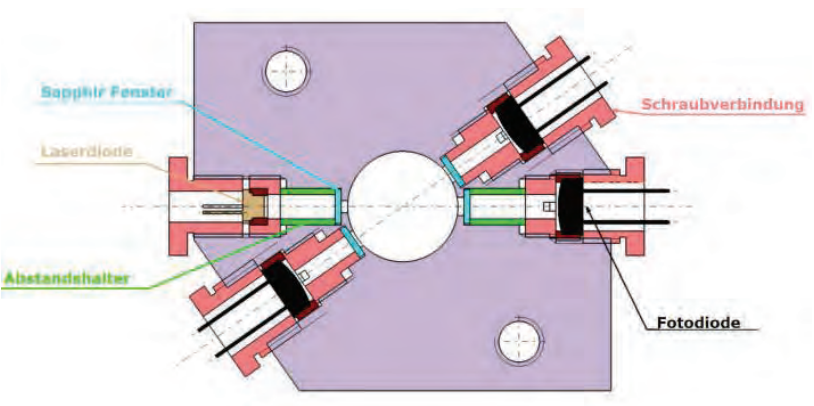

Bild 3 Schnitt durch das Partikelanalysesystem.

\section{Kombiniertes System}

Um ein System zu realisieren, welches chemisch die Ölqualität und gleichzeitig die Partikelbelastung erfasst, wurden beide Sensorsysteme, wie in Bild $\mathbf{4}$ dargestellt, in einem kompakten Gehäuse kombiniert. Die zuvor vorgestellten Systeme sind modular aufgebaut, sodass beide Systeme unabhängig voneinander betrieben werden können. Die Kopplung beider Systeme generiert jedoch einen Mehrwert, da durch den Vergleich der Transmissionskanäle beider Subsysteme eine Selbstüberwachung des kombinierten Systems ermöglicht wird. Zudem besitzt das kombinierte Sensorsystem einen Pt-1000 Temperatursensor, um die Fluidtemperatur im System zu bestimmen. Ausgehend von der gemessenen Temperatur und dem Druckabfall über dem System bei konstantem Fluss kann mit dem System zusätzlich die kinematische Viskosität des Fluides in guter Übereinstimmung mit Referenzmethoden bestimmt werden [7, 8].

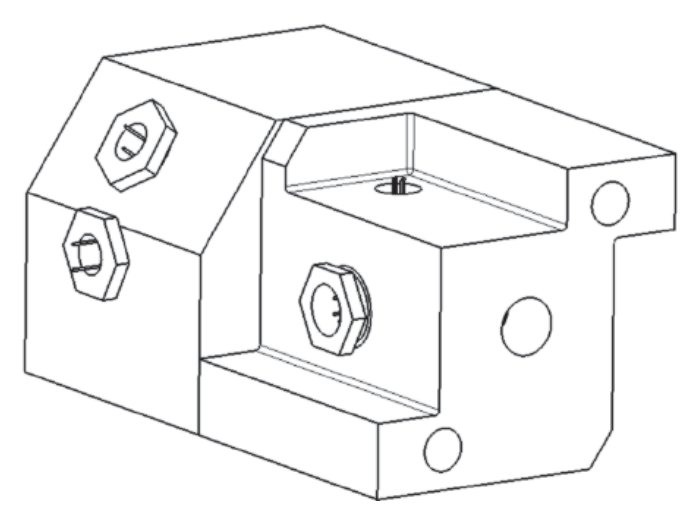

Bild 4 Isometrische Ansicht des kombinierten Ölqualitäts- und Partikelmesssystems.

\section{Ergebnisse}

\section{Ölqualitätssensor}

Zur Durchführung der Untersuchungen mit dem Ölqualitätssensorsystem wurde das synthetische Motorenöl Mobil Pegasus ausgewählt und das Sensorsystem mit vier dazu passenden schmalbandigen Filtern $(5,78 \mu \mathrm{m}, 4,00 \mu \mathrm{m}$ als Referenzkanal, 2,99 $\mu \mathrm{m}$ und 2,86 $\mu \mathrm{m}$ ) ausgestattet („Mineralölkonfiguration“). Das Motorenöl $(1500 \mathrm{ml})$ wurde in einem Ölreaktor bei einer Temperatur von $160{ }^{\circ} \mathrm{C}$ und $50 \mathrm{ml} / \mathrm{min}$ Luftzugabe künstlich gealtert. Nach 0 , 1, 3, 5, 7 und 9 Tagen wurden jeweils Ölproben entnommen und mit einem Labor-FTIRSpektrometer charakterisiert. Die Ergebnisse der spektralen Untersuchung sind in Bild $\mathbf{5}$ dargestellt. Zusätzlich sind die vier Thermopile (TP) -Kanäle des Ölqualitätssensors als gelbe Bereiche markiert. Im Spektrum zeigt die Carbonylbande bei $5,78 \mu \mathrm{m}$ eine starke alterungsbedingte Abnahme der Transmission bedingt durch die Oxidation. Die entnommenen Ölproben wurden anschließend mit dem Sensorsystem vermessen.

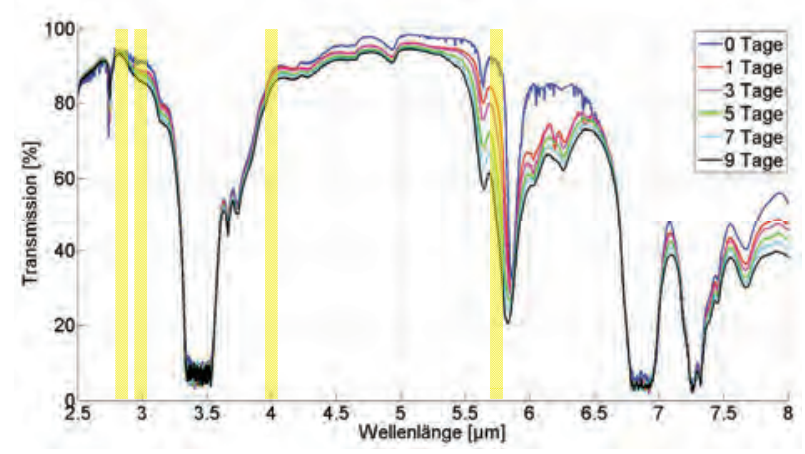

Bild 5 IR-Transmissionsspektren von über 9 Tage bei $160{ }^{\circ} \mathrm{C}$ und $50 \mathrm{ml} / \mathrm{min}$ Luftfluss künstlich gealtertem, synthetischen Motorenöl.

Bild 6 zeigt die normierten DFT-Amplituden aufgetragen über die Dauer der künstlichen Alterung. Um den Einfluss breitbandiger Absorption, insbesondere die erhebliche 
Schwärzung des Öls durch Ruß, zu unterdrücken, wurden die Messkanäle TP1, TP3 und TP4 mit dem Referenzkanal TP2 gewichtet. Auch mit dem Sensorsystem zeigt die deutliche Abnahme des Signals bei TP1 (Carbonylbande) um $>40 \%$ die starke Oxidation während der künstlichen Alterung. Der Referenzkanal TP2 spiegelt die zunehmende Trübung wieder. Die beiden weiteren Kanäle zeigen hier keine Reaktion und können z.B. zur Erkennung von Wasser im Öl oder zur Messung der Abnahme von Additiven genutzt werden. In Bild 7 wird beispielhaft die Reaktion auf Wasserzugabe in synthetischem Motorenöl gezeigt. Sowohl TP3 als auch TP4 zeigen eine starke Abnahme bei zunehmendem Wassergehalt im relevanten Konzentrationsbereich.

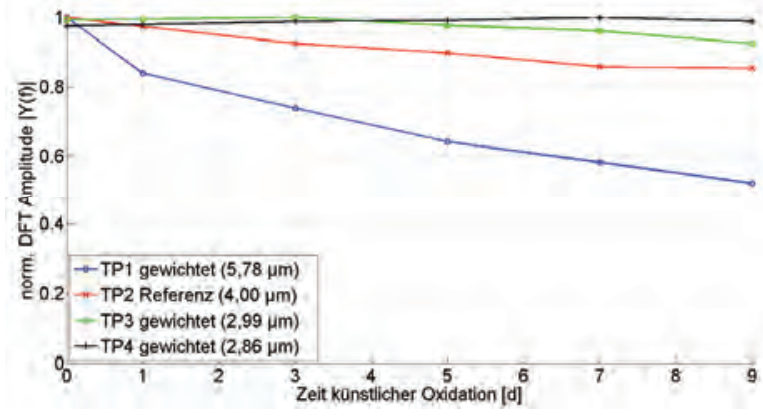

Bild 6 Beispielhafte Messergebnisse zur Bestimmung der Ölqualität für künstlich gealtertes synthetisches Öl (Mobil Pegasus).

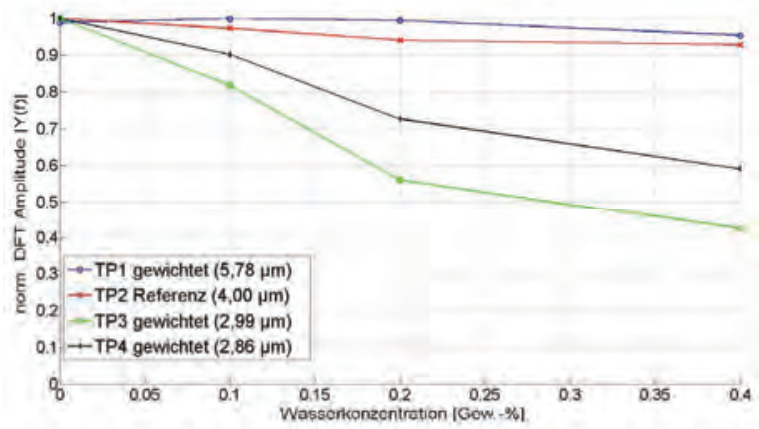

Bild 7 Untersuchung von synthetischem Motorenöl bei Zugabe von Wasser.

In Bild 8 sind verschiedene Schmierflüssigkeiten auf Kohlenwasserstoffbasis nach künstlicher Oxidation sowie Wasserzugabe im 3DDiagramm dargestellt. Es sind jeweils die gewichteten Messkanäle dargestellt. Neben den Hauptbewegungen entlang der TP1-Achse bei künstlicher Oxidation und dem zusätzlichen Abfall bei den Messkanälen TP3 und TP4 in einer Realprobe aus einem Blockheizkraftwerk, vermutlich hervorgerufen durch Blow-by Produkte, zeigt sich bei Wasserzugabe ein starker Abfall der DFT-Amplitude der Messkanäle TP3 und TP4. Der Verlauf bei Wasserzugabe und durch Blow-by Produkte kann nicht ausreichend getrennt werden, sodass hierzu eine Optimierung der Messkanäle und weitergehende Untersuchungen notwendig sind.

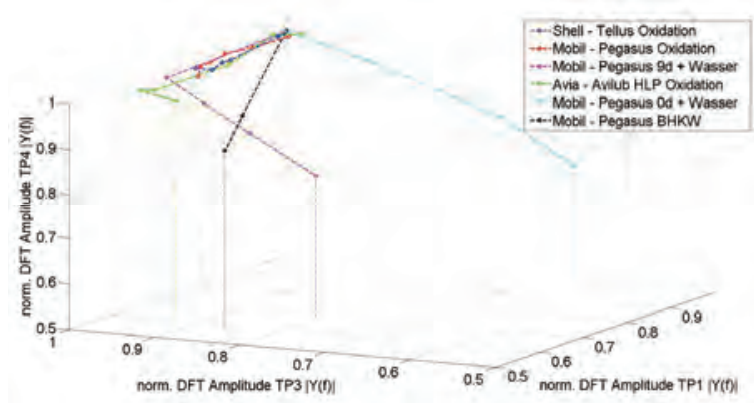

Bild 8 Vergleich unterschiedlicher Schmierflüssigkeiten bei künstlicher Oxidation und Wasserzugabe (Mineralölkonfiguration) mit einer Realprobe aus einem Blockheizkraftwerk (BHKW).

\section{Feldtest zur Bestimmung der Ölqualität}

Das Ölqualitätsmesssystem wurde in eine Mobilplattform integriert und als Feldtestsystem an einer Schraubenpresse erprobt. Hierbei wird der Kühlschmierstoff in der Anlage überwacht, der die während des Pressprozesses entstehende Hitze abführt und für den benötigten Schmierfilm sorgt. Die Mobilplattform wurde mit IR-Filtern in Esterkonfiguration ausgestattet [8] und über einen Zulauf- und Rücklaufschlauch an den Kühlschmierstofftank angeschossen. In Bild 9 sind die Messergebnisse von Thermopile 2 (Wellenlänge 2,75 $\mu \mathrm{m}$ ) über die circa zweimonatige Datenaufzeichnung der Fluidqualität an der Schraubenpresse zu sehen. Weiterhin sind in Bild 9 schwarz gestrichelt die dokumentierten Additivnachfüllungen bzw. Wechsel des Kühlschmierstoffes dargestellt und in Rot das über die vorherigen 50 Datenpunkte geglättete Signal. Aufgrund einiger Systemausfälle sind Sprünge auf der Zeitachse in den Ergebnissen zu sehen. Zu beobachten ist ein starker Anstieg der DFTAmplitude bei Nachfüllen des Kühlschmierstoffes sowie bei der Zugabe von Additiven. Dazwischen ist überwiegend ein kontinuierlicher Abfall des Signals zu beobachten. Die abfallende DFT Amplitude korreliert mit der Belastung des Kühlschmierstoffes und der daraus resultierenden Abnahme des Additivs. Darüber hinaus konnte mit dem Feldtestsystem eine nicht dokumentierte Nachfüllung (Tag 32) erkannt werden. An Tag 26 wurde nur ein Teil (150 I) des Kühlschmierstoffes ausgetauscht, was dazu führt, das weniger unbelasteter Kühlschmierstoff der üblichen Belastung ausgesetzt ist, sodass die DFT-Amplitude schneller abfällt. Nach einem vollständigen Wechsel des Kühlschmierstoffes ist hingegen 
ein geringerer Gradient der DFT-Amplitude zu beobachten. Weiterhin zeigt sich, dass manuelle Eingriffe, wie z.B. das Nachfüllen des Kühlschmierstoffes, bei einer DFT Amplitude von 0,9 durchgeführt werden [8].

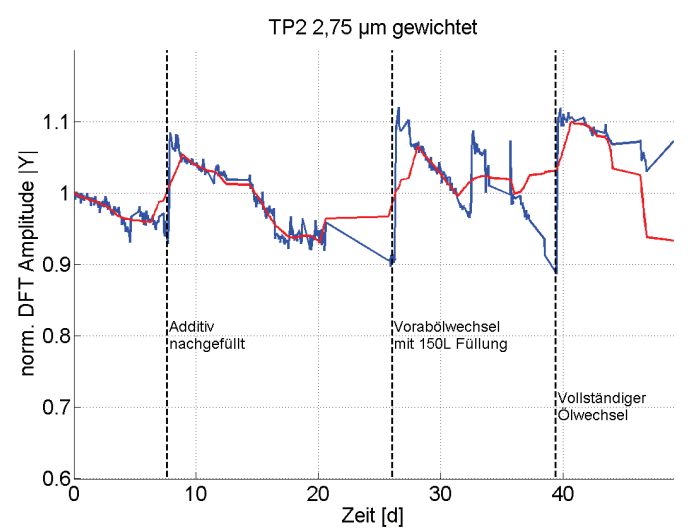

Bild 9 Normierte DFT-Amplitude bei Einsatz der Mobilplattform zur Überwachung von Kühlschmierstoff im Schraubenherstellungsprozess (Esterkonfiguration, TP2 bei 2,75 $\mu \mathrm{m}$ ) [8].

Ausgehend von den erhaltenen Messergebnissen ist nicht nur eine Beurteilung des Verschleißes des Kühlschmierstoffes möglich, sondern bei gleichbleibender Belastung auch eine Prognose bis zum nächsten Nachfüllen des Kühlschmierstoffes bzw. eine Automatisierung dieses Prozesses mit dem Sensorsystem.

\section{Partikelsensor}

Für reproduzierbare Versuche wurden über eine semipermeable Membran und einen Massenflussregler (MFC) Luftblasen in einen Fluidkreislauf injiziert. Um die Unterscheidbarkeit unterschiedlicher Partikel untersuchen zu können, wurde über einen Tank dem Fluidkreislauf verschiedene Mischungen zugegeben: zum einen Öl und Teststaub $(0,2 \mathrm{~g}$ ISO MTD A2 auf $200 \mathrm{ml}$ Öl), zum anderen Öl und Metallpartikel $(1,7 \mathrm{~g}$ auf $200 \mathrm{ml}$ Öl). Jeweils $5 \mathrm{ml}$ beider Mischungen sowie Luftblasen wurden wiederholt in einen Kreislauf mit $500 \mathrm{ml}$ Öl hinzugegeben, so dass durch die Zugabe von $5 \mathrm{ml}$ Staubpartikel-Mischung die Verschmutzungsdichte im System um jeweils $10 \mu \mathrm{g} / \mathrm{ml}$ Staubpartikel (SP) und durch die Zugabe von $5 \mathrm{ml}$ Metallpartikel-Mischung die Verschmutzungsdichte um jeweils $85 \mu \mathrm{g} / \mathrm{ml}$ Metallpartikel (MP) erhöht wurde.

Bild 10 zeigt die resultierenden Messergebnisse. Dargestellt sind die Rückwärts- und Vorwärtsstreuung sowie das Streuverhältnis über die Zeit. Hierbei zeigt sich bei Zugabe von Metallpartikeln primär eine Abnahme der Rückwärtsstreuung, bei Zugabe von Staubpartikeln dagegen ein Anstieg der Vorwärtsstreuung. Die
Luftblasen können durch die scharfen Peaks in allen Signalen identifiziert werden. Filterung des Öles mit einem $1 \mu \mathrm{m}$ Partikelfilter ab $\mathrm{t}=500 \mathrm{~s}$ zeigt in Bild 10 ein Zurücklaufen der Signale auf den Ausgangswert.

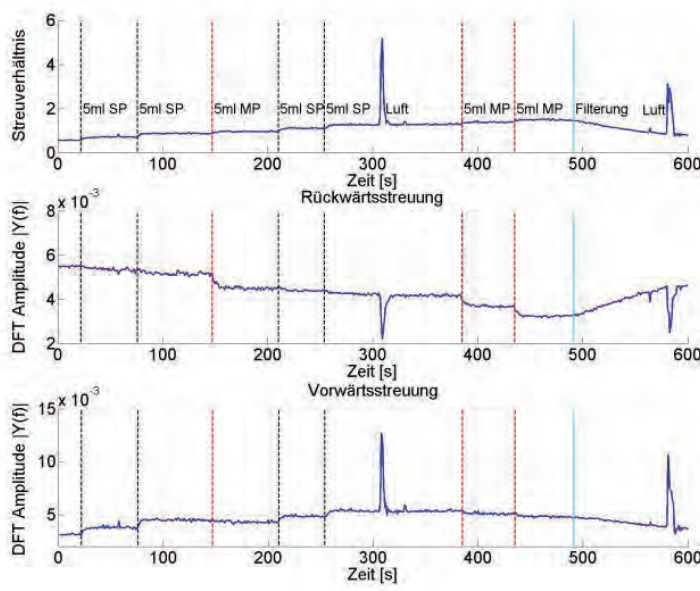

Bild 10 Darstellung des über die Zeit aufgenommenen Streuverhältnisses sowie der Vorwärtsund Rückwärtsstreuung bei Zugabe von Staub- (SP) bzw. Metallpartikeln (MP) und Luftblasen (Luft).

Das Streuverhältnis steigt bei jeder Zugabe von Partikeln an, sodass damit eine Aussage über die Gesamtpartikelbelastung im System getroffen werden kann. Durch Auftragen des Signals der Vorwärtsstreuung über der Rückwärtsstreuung, wie in Bild 11 dargestellt, kann zwischen Metall- und Staubpartikel unterschieden bzw. ihre jeweilige Konzentration bestimmt werden. Eine Zugabe von Staubpartikeln bewirkt primär eine Verschiebung der Signale nach rechts, eine Zugabe von Metallpartikeln primär eine Verschiebung nach unten. Luftblasen zeigen kein konstantes Signal. Wird das Fluid im System gefiltert, zeigt sich auch hier ein Zurücklaufen auf den Ausgangswert.

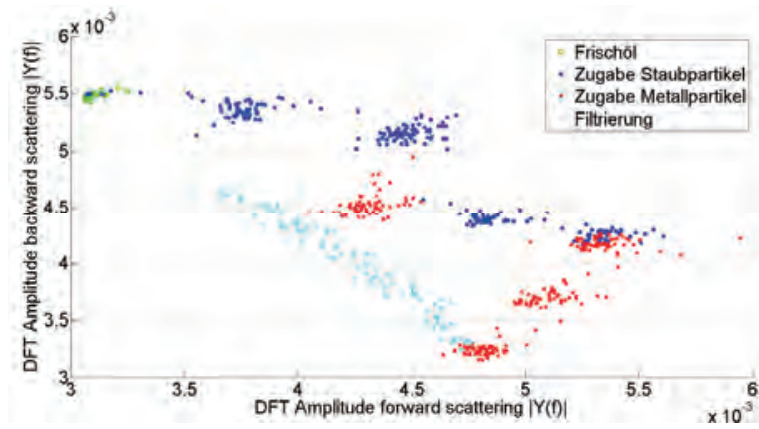

Bild 11 Vorwärtsstreuung aufgetragen gegen Rückwärtsstreuung (DFT-Amplituden). Zugabe von Staubpartikeln bewirkt eine Verschiebung nach rechts, Zugabe von Metallpartikeln nach unten und Filtrierung ein Zurücklaufen auf den Ausgangswert. 


\section{Zusammenfassung}

Es wurde ein kombiniertes Ölqualitäts- und Partikelmesssystem vorgestellt. Mit dem Ölqualitätsmesssystem ist es möglich, Ölalterung, z.B. durch Oxidation oder Blow-byProdukte, sowie den Wassergehalt von Öl zu überwachen. Weiterhin konnte der Verschleiß des Kühlschmierstoffes einer Schraubenpresse überwacht werden und bei gleichbleibender Belastung der nächste Fluidaustausch prognostiziert werden. Mit dem Partikelmesssystem lassen sich Verunreinigungen charakterisieren und quantifizieren sowie die Filterung des Öls kontrollieren. Zusätzlich eröffnet die Betrachtung der redundanten Transmissionskanäle beider Messsysteme das Potential für eine einfache Selbstüberwachung des Gesamtsystems.

\section{Danksagung}

Die zugrunde liegenden Arbeiten wurden teilweise vom Bundesministerium für Bildung und Forschung (BMBF) im Rahmen des Projektes NaMiFlu (FKZ: 16SV5361) im Programm Mikrosystemtechnik sowie vom Land Saarland im Rahmen des ZeMA-Leitprojektes FluidSens gefördert. Weiterhin danken wir RUIA Global Fasteners, Standort Beckingen, für die Ermöglichung des Feldtestes an der Schraubenpresse und für die hierbei geleistete Unterstützung.

\section{Literatur}

[1] T. Bley, A. Schütze: "A Multichannel IR Sensor System for Condition Monitoring of Technical Fluids", Proc. IRS $2011-12^{\text {th }}$ International Conf. on Infrared Sensors and Systems, Nürnberg, June 7 - 8, 2011, DOI: 10.5162/irs11/i4.3

[2] J. Staab: „Industrielle Gasanalyse“, Oldenbourg, erste Auflage, 1994, ISBN 3835628089.

[3] D. Schön, M. Löffler-Mang: „An Optical Sensor for Monitoring Fluid Parameter", $11^{\text {th }}$ International Workshop on Research and Education in Mechatronics, Ostrava, Czech Republic, Sep 910, 2010.

[4] T. Bley, E. Pignanelli, A. Schütze: "COPS Combined Oil quality and Particle measurement System", Proc. ICST $2012-6^{\text {th }}$ International Conf. on Sensing Technology, Kolkata, India, Dec. 18 - 21, 2012.

[5] C. Bohren, D. Huffmann: "Absorption and Scattering of Light by Small Particles", John Wiley \& Sons, 1983, ISBN 0471293407.

[6] D.A. Burns, E.W. Ciurczak: „Handbook of NearInfrared Analysis“, $3^{\text {rd }}$ ed., CRC Press, 2008, ISBN 084937393X.

[7] T. Bley, et al.: "IR Optical Oil Quality Sensor System for High Pressure Applications", in: R. Scheidl, B. Jacoby (Eds.): Mechatronics 2012 - 13th Mechatronics Forum International Conf. Linz, Austria, Sep. 17-19, 2012, Proc. Vol. 2/3, ISBN 978-3-99033-046-5, pp. 351-358.

[8] T. Bley: „Integriertes Multisensorsystem zur Zustandsüberwachung von Schmierflüssigkeiten", Dissertation, Universität des Saarlandes, Shaker Verlag, 2013, ISBN 978-3-8440-2198-1. 